Results Between Feb 2011-Aug 2020, 75 (M=43) patients were treated. Mean age at follow-up was 87.5 months (range 18-157 m). 42 cases were bilateral and thus 117 feet were treated. $45(60 \%)$ were idiopathic and 30 (40\%) nonidiopathic.

Of the 75 patients, 50 (67\%) underwent tenotomy under local anaesthetic in clinic. Tenotomy rates were higher in nonidiopathic vs idiopathic patients $(83 \%$ vs $56 \%)(\mathrm{P}<0.002)$

Mean dorsiflexion (knee extended) in affected feet was $14^{\circ}$. In unilateral cases mean dorsiflexion (knee extended) in unaffected foot vs affected foot was $17^{\circ}$ vs $12^{\circ}$. All idiopathic feet were plantigrade.

Abnormal evertor function was seen in 3 of 66 (5\%) idiopathic feet versus 22 of 51 non-idiopathic feet $(43 \%)$ $(\mathrm{p}<0.0001)$.

Calf circumference discrepancy was higher in unilateral cases versus bilateral (mean $1.3 \mathrm{~cm}$ versus $0.6 \mathrm{~cm}$ respectively). Unilateral cases undergoing tenotomy had a greater mean circumference difference $(1.5 \mathrm{~cm})$ versus those managed without tenotomy $(0.7 \mathrm{~cm})$.

43 idiopathic patients were suitable for OXFORD scoring at time of last review (i.e. older than 5 years old). The overall median OxFAQ was $96 \%$ with points lost only in the physical scale. Function in non-idiopathic cases was determined by their overall condition.

Conclusion The idiopathic club foot is well treated by a physiotherapy-delivered Ponseti technique with excellent outcomes at $5 \mathrm{yr}$ follow-up both subjectively and objectively.

\section{COMPARISON OF FUNCTIONAL ABILITY IN SIBLINGS WITH DUCHENNE MUSCULAR DYSTROPHY}

${ }^{1}$ Catherine Rye, ${ }^{2}$ Marion Main, ${ }^{3}$ Francesco Muntoni. ' Great Ormond Street Hospital and University College London; ${ }^{2}$ Great Ormond Street Hospital NHS Foundation Trust; ${ }^{3}$ UCL Great Ormond Street Institute of Child Health

10.1136/archdischild-2020-gosh.87

Early diagnosis is important to optimise management and prolong function in patients with Duchenne Muscular Dystrophy (DMD). There is little research comparing functional outcomes of siblings diagnosed at different ages.

This study aims to analyse the effect of age at diagnosis and prescription of steroids on function in siblings with the same genetic diagnosis of DMD. Functional ability was measured using the North Star Ambulatory Assessment (NSAA) which is a validated outcome measure in ambulant children with DMD.

NSAA data from 24 siblings (48 patients) was collected from their first NSAA assessment at Great Ormond Street Hospital, including all scores until July 2020.

Age matched NSAA scores were collated at 6 monthly intervals ( \pm 3 months) between 5.5-10.5 years in siblings, where one or more NSAA scores could be compared. 17 siblings (34 patients) met the inclusion criteria and were analysed.

Results show that younger siblings were diagnosed on average 2.7 years earlier and started steroids 0.8 years earlier. The median peak NSAA scores were higher in older siblings at ages 5.26-6.25 and 7.26-8.25 years. Between 6.26-7.25 and after 8.26 years, the younger siblings median NSAA scores were consistently higher, although there were small numbers in each sub-group. Wilcoxon Signed Rank test showed no significant differences between groups.

Analysis of the graph suggests that despite the earlier age at diagnosis, younger siblings did not diverge from the older sibling in the first phase of the disease. Visual inspection of the NSAA score shows that after 8 years old, the younger siblings consistently scored higher values. This suggests that earlier initiation of steroids is likely to have played a role in this outcome, as previously demonstrated in the DMD population. Further research will assess long-term effects of these trends, regarding age at loss of ambulation, and of respiratory insufficiency.

\section{THE RELATIONSHIP BETWEEN UPPER AND LOWER LIMB FUNCTION IN A COHORT OF CHILDREN WITH CHARCOT- MARIE-TOOTH DISEASE}

${ }^{1}$ Evelin Milev, ${ }^{2}$ Matilde Laura, ${ }^{3}$ Sarah Rand, ${ }^{3}$ Melissa Walk-Ley, ${ }^{1}$ Amy Wolfe, ${ }^{1}$ Catherine Rye, ${ }^{2}$ Mary Reilly, ${ }^{1}$ Francesco Muntoni. ${ }^{1}$ UCL Great Ormond Street Institute of Child Health; ${ }^{2}$ Queen Square Centre for Neuromuscular Disease, UCL; ${ }^{3}$ UCL

\subsection{6/archdischild-2020-gosh.88}

Charcot-Marie-Tooth (CMT) is a progressive disease with clinical signs presenting first in the distal lower extremities. Upper limb function in this population is also affected at a later stage of life but it is poorly researched and little is known about hand function limitations and loss of manual dexterity. The purpose of this study is to investigate the possible relationship between upper and lower limb function in a group of children and adolescents. The CMT natural history study at Great Ormond Street Hospital in London has been collecting longitudinal data of more than 120 children and adolescents with CMT (age range 4 to 21 years). Eighty one children with CMT type 1 (53\% CMT1A), 22 with type 2 and 21 with other types of CMT have been assessed up to date. To evaluate upper limb function we used myometry, functional dexterity test and 9 hole peg test; to measure lower limb function the 6 minute walk test, long jump and plantar and dorsiflexion strength tests. In this study we will use correlation analysis to explore the concordance between the upper and the lower limb function. A comparison will be made between children and adolescents with demyelinating CMT and those with axonal CMT, and also individual genotypes, to look if variation in genetic subtype affects individuals in different ways. In our analysis we will assess longitudinally the correlation between upper and lower limb function in children with different subtypes of CMT. If a strong relationship between the two is found in individual conditions, we will assess further the predictive model of loss of function between upper and lower limbs in different genetically defined conditions. This study is a part of a MSc project being undertaken at UCL and a full report and results will be presented later in the year.

\section{CHRONIC DISSEMINATED CANDIDIASIS TREATED WITH ADJUVANT CORTICOSTEROID TREATMENT}

Srdan Rogosic, Charlotte Patterson, Jack Bartram, Vesna Pavasovic-Jovanovic Sujith Samarasinghe, Philip Ancliff, Anupama Rao, David O'Connor, Ajay Vora, Sara Ghorashian. Department of Haematology, Great Ormond Street Hospital for Children NHS Foundation Trust

10.1136/archdischild-2020-gosh.89 
Background Chronic disseminated candidiasis (CDC) can be a severe infection in immunocompromised patients. CDC/IRIS is an associated immune reconstitution syndrome most commonly characterised by prolonged fever despite adequate antimicrobial cover, raised inflammatory markers and typical radiological changes, often occurring during neutrophil recovery and causing significant morbidity in itself.

Objective To establish the outcomes of corticosteroid use in CDC/IRIS patients.

Methods In this retrospective study, we included all the patients with CDC/IRIS treated with corticosteroids in our Department from March 2017 until May 2020. We defined CDC/IRIS with persistent fevers, resistant to a minimum of 2 weeks of antifungal treatment, raised inflammatory markers and accompanying radiological changes. The data was collected through searches in the laboratory system and the medical notes.

Results 6 patients fit the criteria for CDC/IRIS. All the patients received a substantial course of antifungals before starting corticosteroids (median 47.5 days (range 15-107)). All patients had raised CRP (median 186 (range 77-271)). 5 patients had positive radiological changes in the spleen, liver or kidney. 1 patient only had pulmonary changes. The duration of fevers prior to starting corticosteroids was 46 days (range 21-96).

Most patients (5/6) received a short course of methylprednisolone, dexamethasone or hydrocortisone, followed by prednisolone for the rest of the treatment. The median duration of corticosteroid treatment was 94 days (range 34-400).

Fever resolved on the same day or the day after starting corticosteroids in 5/6 patient. 5/6 patients remained apyrexial more than seven days after stopping corticosteroids.

Conclusion These findings confirm that adjuvant corticosteroids are helpful in the management of CDC/IRIS. However, prolonged corticosteroid treatment has associated complications and can impair anti-fungal immune responses so its use has to be judged carefully. In this series, all patients derived clinical benefit and there were no cases of clinical deterioration related to progression of fungal infection.

\section{RAPID EXOME SEQUENCING SERVICE FOR ACUTELY UNWELL CHILDREN WITH A LIKELY MONOGENIC DISORDER}

${ }^{1}$ Edward Steel, ${ }^{2}$ Harry Leitch, ${ }^{2}$ Jan Cobben, ${ }^{1}$ Emma Wakeling. ${ }^{1}$ Great Ormond Street Hospital; ${ }^{2}$ Northwick Park Hospital

10.1136/archdischild-2020-gosh.90

The Rapid exome sequencing service for acutely unwell children with a likely monogenic disorder was launched by NHS England in October 2019. The aim of this new service is to facilitate the rapid diagnosis of genetic conditions in paediatric patients in intensive care settings where a single gene disorder is likely and diagnosis is expected to result in an immediate change in patient management. Funding is provided for recruitment of up to 700 patients per year via seven Genomic Medicine Services (GMS) across England.

Trio (patient and both parents) exome sequencing is offered for all referrals accepted by the testing laboratory in Exeter. Patient and parent exomes are compared to identify variants in single genes which may point to a diagnosis. Results are returned to the referring department with a target turnaround time of 21 days.

All referrals from clinicians within the region covered by the London North GMS are reviewed by the North East or North West Regional Genetics Services (based at GOSH and Northwick Park Hospital, respectively) to ensure that patients meet eligibility criteria. The service is targeted toward patients in neonatal and paediatric intensive care units (NICU/PICU). However acutely unwell patients in other settings are accepted on a case-by-case basis, as are unwell or deceased patients where there is a time-sensitive risk of recurrence in a current pregnancy. Reasons for rejection include a diagnosis being unlikely to impact management, a likely environmental or polygenic cause, and more specific or appropriate methods of testing being available.

We present the uptake and outcomes of the service across the London North region in the first year of recruitment.

\section{GREAT ORMOND STREET HOSPITAL SUMMER SCHOOL 2020 - EMBRACING THE \#DIGITALLIFE IN CONFERENCING}

${ }^{1}$ Abbie Tutt, ${ }^{2}$ Shuang Liu, ${ }^{2}$ Jonathan Smith, ${ }^{2}$ Justin Poisson, ${ }^{2}$ Simon Blackburn, ${ }^{2}$ Marissa Willock, ${ }^{2}$ Jeroen Poisson, ${ }^{2}$ Nicola Palmer, ${ }^{2}$ Shereen Brown, ${ }^{2}$ Olivia Wheeler, ${ }^{2}$ Peter Stow, ${ }^{2}$ Ellie McGonegal, ${ }^{2}$ Cristine Sortica da Costa. ${ }^{1}$ Warwick University; ${ }^{2} P G M E$, Great Ormond Street Hospital

\subsection{6/archdischild-2020-gosh.91}

Background Social Media is an accompanying staple to conferences but also allows attendees who are attending virtually to interact with the day. GOSH Summer School (GSS) is normally run as a face to face event but had to be moved to a virtual set up this year due to the impact of Covid-19. This forced our attendees to change the way they networked and interacted with the day and how we dispersed education to the attendees.

Methods Two main accounts were used to document the day; Twitter: @GOSHPGME and Instagram: @pgme.education. Positive feedback, attendees 'set ups' and screenshots (in replace of taking photos) of the talks were shared. Attendees were encouraged to use the hashtag \#GoshSummerSchool or send photos to GOSH PGME Instagram.

Results The PGME Twitter account gained 155 followers and was mentioned 196 times. This resulted in 2351 profile visits to the account and overall, $56.1 \mathrm{~K}$ impressions were made throughout GSS. On Instagram, 24 posts were made and gained 77 new followers. There was an average of 30.5 likes, 0.5 comments, 3.9 post sends, 5.9 post saves, 26.6 profile visits and 436 views per post. 50 posts per day were added to the story feature of Instagram mainly featuring attendee set ups and opinions of the talks given. During the GSS, a survey about which additional resources students use to gain knowledge in medicine, social medial received $48 \%$ votes.

Discussion The engagement statistics and broad use of social media to compliment the GSS shows how it can be used to increase engagement on virtual conferences. The uptake of social media interaction shows the potential of the platform to enable increased engagement and sharing of medical education. This also enables the increase of long-distance attendees allowing GOSH to open medical education beyond London schools and even worldwide. 